\title{
Erratum: Systematic Investigation of Anisotropic Magneto-Peltier Effect and Anomalous Ettingshausen Effect in Ni Thin films [Phys. Rev. Applied 11, 034022 (2019)]
}

\author{
Raja Das, Ryo Iguchi, and Ken-ichi Uchida ${ }^{*}$
}

(Received 22 July 2019; published 6 August 2019)

DOI: 10.1103/PhysRevApplied.12.029901

We identified an error in Fig. 3(b) in the original article, where the $\phi_{\text {AMPE }}$ image for $\theta_{H}=90^{\circ}$ was replaced by that for $\theta_{H}=0^{\circ}$. As shown below, we have corrected the error. All the conclusions in the original article remain unaffected. Since the analyzed $\theta_{H}$ dependence of $\Delta T_{\mathrm{AMPE}}$ in the original figure is correct and consistent with the previous results shown in Ref. [1], Fig. 3(e) is not replaced in the revised figure.
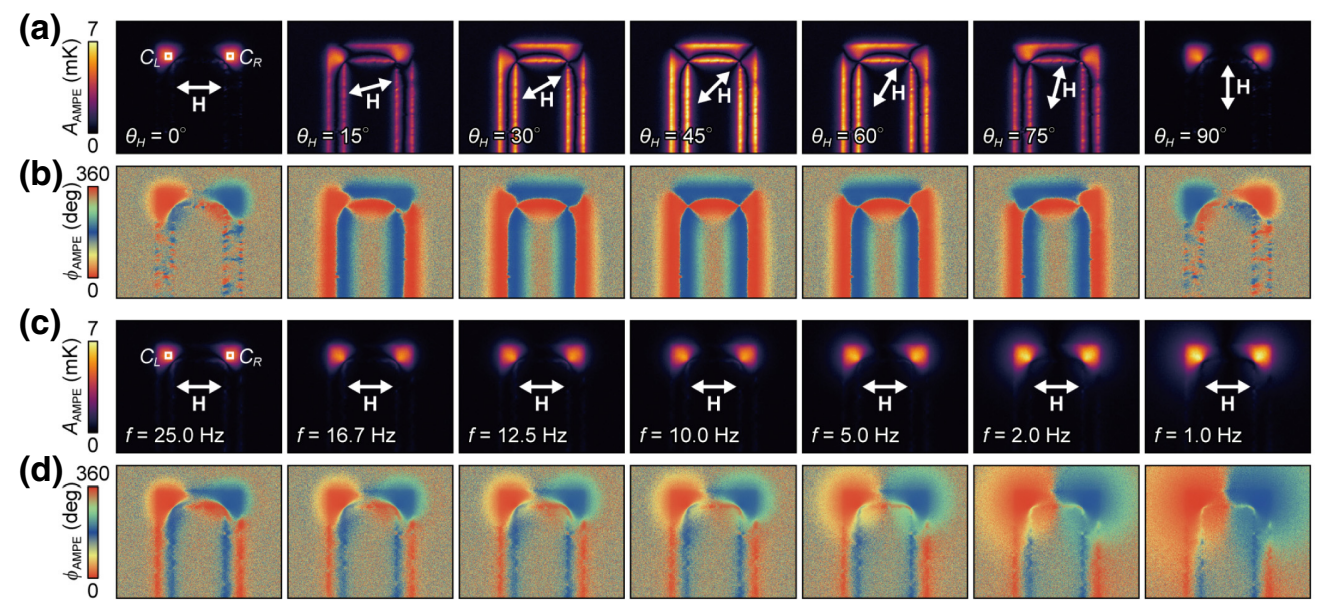

(e)
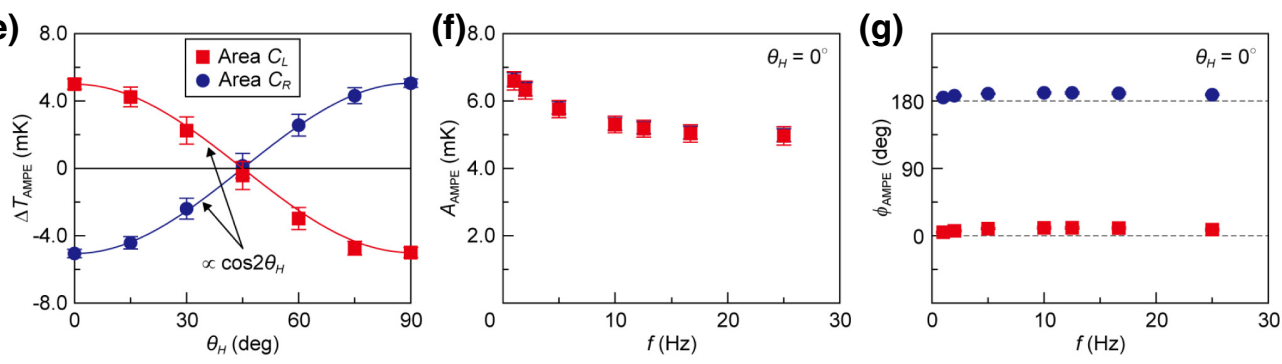

FIG. 3. (a),(b) $A_{\mathrm{AMPE}}$ and $\phi_{\mathrm{AMPE}}$ images for the U-shaped 100 -nm-thick Ni film on the glass substrate for various values of $\theta_{H}$, measured at $J_{c}=40 \mathrm{~mA}$ and $f=25 \mathrm{~Hz}$. (c),(d) $A_{\mathrm{AMPE}}$ and $\phi_{\mathrm{AMPE}}$ images for the U-shaped 100-nm-thick Ni film on the glass substrate for various values of $f$, measured at $\theta_{H}=0^{\circ}$ and $J_{c}=40 \mathrm{~mA}$. (e) $\theta_{H}$ dependence of $\Delta T_{\mathrm{AMPE}}$ on the area $C_{L / R}$ for the $U$-shaped 100 -nm-thick Ni film on the glass substrate at $J_{c}=40 \mathrm{~mA}$ and $f=25 \mathrm{~Hz}$. The solid curves show the cos $2 \theta_{H}$ functions for comparison with the experimental results. (f),(g) $f$ dependence of $A_{\text {AMPE }}$ and $\phi_{\text {AMPE }}$ on $C_{L / R}$ for the U-shaped 100-nm-thick Ni film on the glass substrate, measured at $\theta_{H}=0^{\circ}$ and $J_{c}=40 \mathrm{~mA}$. The data points in (e)-(g) are obtained by our averaging the $A_{\mathrm{AMPE}}$ and $\phi_{\mathrm{AMPE}}$ values on $C_{L / R}$ defined by the squares in (a),(c). The error bars represent the standard deviation of the data in the corresponding squares.

[1] K. Uchida, S. Daimon, R. Iguchi, and E. Saitoh, Observation of anisotropic magneto-Peltier effect in nickel, Nature 588, 95 (2018).

*uchida.kenichi@nims.go.jp 